\title{
Ajuste a un modelo matemático, comparación de las curvas de crecimiento y características morfológicas de cuatro Urochloas de una colección in vivo establecida en Antioquia, Colombia
}

\author{
Adjustment to a mathematical model, comparison of the growth curves and \\ morphological characteristics of four Urochloas from an in vivo collection \\ established in Antioquia, Colombia
}

\author{
Manuela Ortega-Monsalve ${ }^{1,3}$, Andrés Mateo Velásquez-Henao ${ }^{1}$, \\ Alejandro Ortiz-Acevedo ${ }^{1}$, Luis Fernando Galeano-Vasco ${ }^{1}$, \\ Marisol Medina-Sierra ${ }^{2}$
}

\section{Resumen}

El objetivo del estudio fue ajustar las curvas de crecimiento de cuatro Urochloas mediante un modelo matemático que permita la comparación entre ellas. Se tuvieron en cuenta las variables de altura $(\mathrm{cm})$, temperatura $\left({ }^{\circ} \mathrm{C}\right)$, precipitación $(\mathrm{mm})$ y edad del pasto (días). El crecimiento de cada especie se analizó en el software estadístico R-Project. Se ajustaron los datos de crecimiento de las especies con el uso del modelo cuadrático yt $=$ $\beta_{0}+\beta_{1} t-\beta_{3} t^{2}$, con coeficientes de determinación $\left(\mathrm{R}^{2}>0.9\right)$ y un valor de $\mathrm{p}<0.001$. Como resultado, Urochloa brizantha cv Piatá presentó un $\mathrm{R}^{2}=0.9859$ y fue la especie más influenciada positivamente en su crecimiento por el efecto de la edad (días), seguido por U. decumbens, U. mutica y U. brizantha cv Toledo, esta última con el menor ajuste. La precipitación influyó en la tasa de crecimiento de la U. mutica mientras que la temperatura

\footnotetext{
${ }^{1}$ Universidad de Antioquia, Facultad de Ciencias Agrarias, Medellín, Colombia

${ }^{2}$ Universidad de Antioquia, Facultad de Ciencias Agrarias, Grupo de Investigación en Agrociencias, Biodiversidad y Tterritorio GAMMA, Medellín, Colombia

${ }^{3}$ E-mail: manuela.ortegam@udea.edu.co
}

Recibido; 11 de febrero de 2021

Aceptado para publicación: 31 de agosto de 2021

Publicado: 27 de octubre de 2021

CLos autores. Este artículo es publicado por la Rev Inv Vet Perú de la Facultad de Medicina Veterinaria, Universidad Nacional Mayor de San Marcos. Este es un artículo de acceso abierto, distribuido bajo los términos de la licencia Creative Commons Atribución 4.0 Internacional (CC BY 4.0) [https:// creativecommons.org/licenses/by/4.0/deed.es] que permite el uso, distribución y reproducción en cualquier medio, siempre que la obra original sea debidamente citada de su fuente original 
no tuvo influencia en el crecimiento de las especies. Las curvas se ajustaron a modelos cuadráticos, lo que indica que su crecimiento puede compararse y predecirse a lo largo del tiempo, lo que facilita su manejo y óptimo aprovechamiento.

Palabras clave: variables agronómicas, crecimiento, pasto, variables climáticas

\section{Abstract}

The aim of this study was to adjust the growth curves of four Urochloas using a mathematical model that allows the inter comparison. The variables of height $(\mathrm{cm})$, air temperature $\left({ }^{\circ} \mathrm{C}\right)$, precipitation $(\mathrm{mm})$ and pasture age (days) were evaluated. The growth of each species was analysed in the statistical software R-Project. The growth data of the species were adjusted with the use of the quadratic model $y t=\beta_{0}+\beta_{1} t-\beta_{3} t^{2}$, with coefficients of determination $\left(\mathrm{R}^{2}>0.9\right)$ and a value of $\mathrm{p}<0.001$. As a result, Urochloa brizantha cv Piatá presented an $\mathrm{R}^{2}=0.9859$ and was the species most positively influenced in its growth by the effect of age (days), followed by $U$. decumbens, U. mutica and $U$. brizantha cv Toledo, the latter with the least adjustment. Precipitation influenced the growth rate of $U$. mutica while temperature had no influence on the growth of the species. The curves were fitted to quadratic models, which indicates that its growth can be compared and predicted over time, which facilitates its management and optimal use.

Key words: agronomic variables, growth, grass, climatic variables

\section{INTRODUCCIÓN}

En Colombia existe una gran variedad de pastos y especies forrajeras nativas que a lo largo del tiempo se han utilizado para la alimentación animal. Sin embargo, con el avance de la tecnología se han introducido especies de pastos mejorados en el país que proporcionan a los productores mejores características de manejo y productividad. Las gramíneas están representadas en Colombia por 907 especies y 183 géneros (Giraldo, 2013). Especies del género Urochloa, antes conocidas como género Brachiaria, son las de más amplia distribución en el país y en general en los sistemas ganaderos del trópico latinoamericano (Rivas y Holmann, 2004).

El género Urochloa pertenece a la tribu Paniceae que es originario del continente africano. Incluye cerca de 100 especies distribuidas en las regiones tropicales (Renvoize et al., 1987). En América tropical se utilizan principalmente siete especies - U. brizantha, $U$. decumbens, $U$. dictyoneura, $U$. humidicola, U. mutica y U. ruziziensis (Argel y Keller-Grein, 1998). Dentro de las Urochloa existen diferencias en su morfología, calidad nutricional, forma de crecimiento y desarrollo. El crecimiento de las plantas es un proceso fisiológico bastante complejo que se ve influenciado por diferentes factores (Mohr, 1995). Según Hunt (2003) y Hunt et al. (1984). Los cultivares Piatá y Toledo presentan grandes ventajas en su establecimiento y producción de biomasa ya que poseen tolerancia al ataque de plagas y enfermedades y una mayor adaptación a los ambientes adversos. Los pastos mejorados poseen las características de mejorar la fertilidad del suelo, aumentar la producción de leche y carne bajo condiciones extremas del suelo (CIAT, 2010). Conocer el crecimiento de las plantas en el momento actual y predecirlo a futuro es una ventaja que proporciona información útil 
para programar actividades de manejo en fincas ganaderas, debido a la importancia del pasto como recurso en la producción (Ramírez et al., 2011). Esta variable también permite conocer las características productivas de las plantas y lograr su interpretación biológica (Torres et al., 2012).

Ajustar el crecimiento a un modelo matemático permite predecir y evaluar diferentes parámetros de interés zootécnico (Freitas, 2005). Los modelos más utilizados para determinar el comportamiento en el crecimiento de los animales y las plantas han sido modelos no lineales como: Brody (Brody 1945), Von Bertalanffy (Bertalanffy 1957), Richards (Richards 1959), Logística (Nelder 1961) y Gompertz (Laird 1965) (Torres et al., 2012). Estos modelos están basados en el crecimiento de seres biológicos y se evalúan según su bondad de ajuste a los datos obtenidos, la interpretación biológica de los parámetros, la dificultad de la aplicación informática y la influencia de los parámetros ambientales sobre la curva de crecimiento (Brown et al., 1976). Los modelos no lineales pueden permitir una mejor interpretación y asociación entre los resultados obtenidos de procesos o fenómenos físicos ya que usan menos parámetros que los modelos lineales, lo que permite tener predicciones más confiables y con una mayor facilidad para interpretar los parámetros que hacen parte de la variable respuesta (Pinheiro y Bates, 2000).

Las curvas de crecimiento proporcionan información de gran valor entre dos o más variables que permiten tomar decisiones como, por ejemplo, cuál es el punto óptimo de corte o pastoreo, porcentaje de forraje verde, materia seca y relación hoja-tallo. Existe un gran desconocimiento acerca de las curvas de crecimiento aplicadas a la producción agropecuaria, lo que limita la implementación de nuevos programas que permitan realizar mejoras en el campo zootécnico y elevar la productividad (Agudelo et al., 2008). La Universidad de Antioquia tiene una colección in vivo de especies forrajeras, dentro de las cuales se encuen- tran especies del género Urochloa. En la investigación, se incluyeron las especies $U$. decumbens, $U$. brizantha cv. Piatá, $U$. brizantha $\mathrm{cv}$. Toledo y U. mutica. Este trabajo tuvo como objetivo ajustar las curvas de crecimiento de las cuatro urochloas mediante un modelo matemático que permita la comparación entre las especies, así como conocer la correlación que existe entre las variables precipitación y temperatura y como influyen en la altura de crecimiento para cada especie.

\section{Materiales y Métodos}

\section{Área de Estudio}

El trabajo se realizó en la colección in vivo de especies forrajeras de la Facultad de Ciencias Agrarias de la Universidad de Antioquia, ubicada en el municipio de Medellín, a $1600 \mathrm{msnm}$. Se seleccionaron cuatro especies de la colección: $U$. decumbens, $U$. mutica, U. brizantha $\mathrm{cv}$ Toledo y U. brizantha cv Piatá. Estas se encuentran en parcelas y son utilizadas por los estudiantes y docentes para realizar diferentes investigaciones.

\section{Composición nutricional}

Se realizó el análisis bromatológico de muestras de las cuatro especies de Urochloa en 2017 por medio del método de espectroscopía de infrarrojo cercano (NIRS) en el laboratorio de Nutrición Animal de la Facultad de Ciencias Agrarias. Se determinó el contenido de materia seca, proteína bruta, fibra detergente neutra (FDN), fibra detergente ácida (FDA), lignina, grasa cruda, cenizas y azúcares.

\section{Recopilación de Datos}

Se recopiló información de trabajos realizados con las Urochloas en la Facultad de Ciencias Agrarias. Estos trabajos contenían diferentes números de réplicas y muestras 


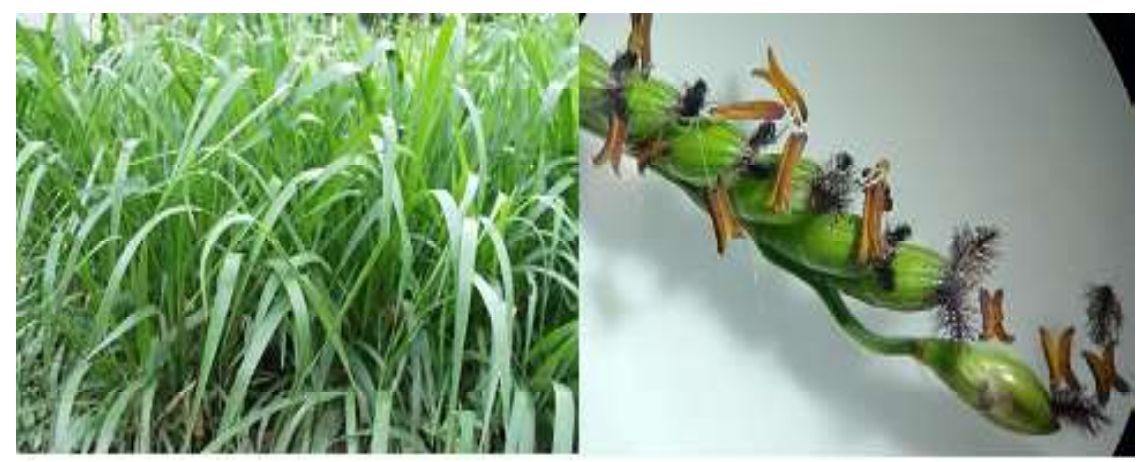

Figura 1. Planta de Urochloa brizantha Cultivar Toledo. (Izquierda) Se observa el desarrollo de los tallos y las macollas. (Derecha) Inflorescencia del pasto Toledo. Se observan las estructuras morfológicas que conforman la espiguilla

de los pastos: U. mutica (9 muestras y 14 réplicas), $U$. brizantha cv Toledo (5 muestras y 5 réplicas), $U$. decumbens (5 muestras y 5 réplicas), y $U$. brizantha cv Piatá (11 muestras y 5 réplicas), los cuales fueron ejecutados entre marzo de junio de 2017 y de septiembre a noviembre de 2018. Para determinar el crecimiento de las plantas se realizaron mediciones semanales. Las variables de precipitación y temperatura fueron obtenidas de la base de datos del SIATA (Sistema de Alerta Temprana del Valle de Aburrá) según el tiempo en que fueron tomadas las mediciones de las especies en estudio.

\section{Curvas de Crecimiento}

Para la realización de las curvas de crecimiento ajustadas a un modelo matemático y la obtención de las correlaciones entre las variables de cada especie se consideraron las variables crecimiento del pasto $(\mathrm{cm})$, edad del pasto (días), y precipitación $(\mathrm{mm})$ y temperatura en la zona $\left({ }^{\circ} \mathrm{C}\right)$. En las gráficas presentadas las variables utilizadas son la edad del pasto (x) y el crecimiento (y). Las variables precipitación y temperatura se utilizaron para determinar su efecto sobre el crecimiento de las plantas y si existía correlación entre ellas.
El crecimiento de cada especie se analizó en el software estadístico R-Project para seleccionar y ajustar el modelo de ecuación que más se acomodó a cada una de ellas. Para realizar este análisis estadístico se utilizó la librería $R c m d r$ contenida en R-Project. Se obtuvieron los valores de las medias, desviaciones estándar y coeficientes de variación para cada variable y para cada especie. En R-Project se ajustaron las curvas de crecimiento de las cuatro especies a modelos matemáticos cuadráticos $y t=\beta_{0}+\beta_{1} t-\beta_{3} t^{2}$ donde $\beta_{0}$ es un valor constante de la variable $\langle\mathrm{y}\rangle$ (crecimiento) cuando $\mathrm{x} y \mathrm{t}$ son cero $\mathrm{y}$ donde $\beta_{1}$ es la pendiente en la cual se muestra los cambios que sufre la variable $\mathrm{x}$ (días) y se mide la relación entre x e y (crecimiento vs. días).

\section{Resultados y Discusión}

\section{Morfología de las Especies y Composi- ción Nutricional}

Se presenta un registro fotográfico de las especies del género Urochloa con el fin de facilitar el reconocimiento y la diferenciación de las estructuras morfológicas.

\section{Análisis de la Información}




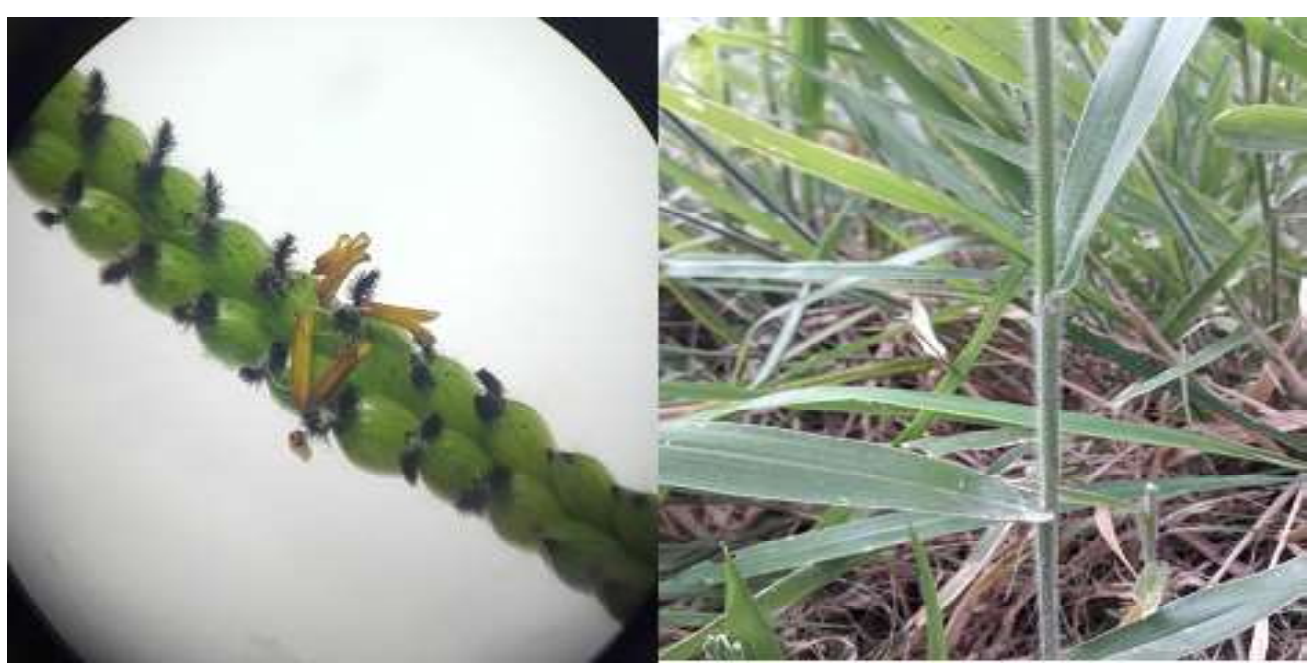

Figura 2. Urochloa decumbens. (Derecha) Tricomas que cubren el tallo y las hojas. (Izquierda) Inflorescencia. Se observan las dos hileras que componen la espiguilla. También se puede ver los filamentos, las anteras y el estigma

Urochloa brizantha (Hochst. Ex A. Rich) R. Webster Cultivar Toledo (CIAT 26110)

Gramínea perenne con crecimiento en forma de macolla que puede alcanzar $1.60 \mathrm{~m}$ de altura. Produce tallos vigorosos capaces de enraizar a partir de nudos cuando entra en contacto con el suelo. Posee hojas lanceoladas con pocas pubescencias que alcanzan hasta $60 \mathrm{~cm}$ de longitud (Peters y Franco, 2011). La inflorescencia es en forma de panícula, cuenta con 4 racimos de 8 a 12 $\mathrm{cm}$ con una sola hilera de espiguillas como se muestra en la Figura 1 (Peters y Franco, 2011).

El pasto Toledo puede alcanzar valores de proteína cruda (PC) entre 8 y $13 \%$, cuando la planta es cosechada entre los 25 a 45 días del rebrote (Lascano et al., 2002). Ortega et al. (2015) reportaron un contenido de fibra detergente neutra (FDN) de 70.78\%, en tanto que Vanegas (2015) al evaluar la composición nutricional encontraron contenidos de materia seca (MD) de $28.04 \%$, FND de $67.27 \%$ y PC de $9.20 \%$. El valor nutricional del pasto depende, indudablemente de las condiciones del suelo y de la época del año.

Urochloa decumbens (Stapf) R.D. Webste

El pasto $U$. decumbens es una gramínea perenne. Posee tallo decumbente, cuenta con raíces fuertes y duras, además de pequeños rizomas. Los tallos van de cilíndricos a ovalados, pueden ser erectos o decumbentes, de color verde y algunas veces con visos morados (Olivera et al., 2006). Posee tricomas en los tallos y las hojas. Su inflorescencia es un racimo floral corto, compuesto por dos hileras (Figura 2).

Las Urochloas poseen características que ayudan a mejorar el rendimiento productivo de las producciones donde se emplean como alimento para los animales (Combatt et al., 2015). U. decumbens es una especie con un alto potencial productivo de MS en suelos pobres gracias a su capacidad de adap- 


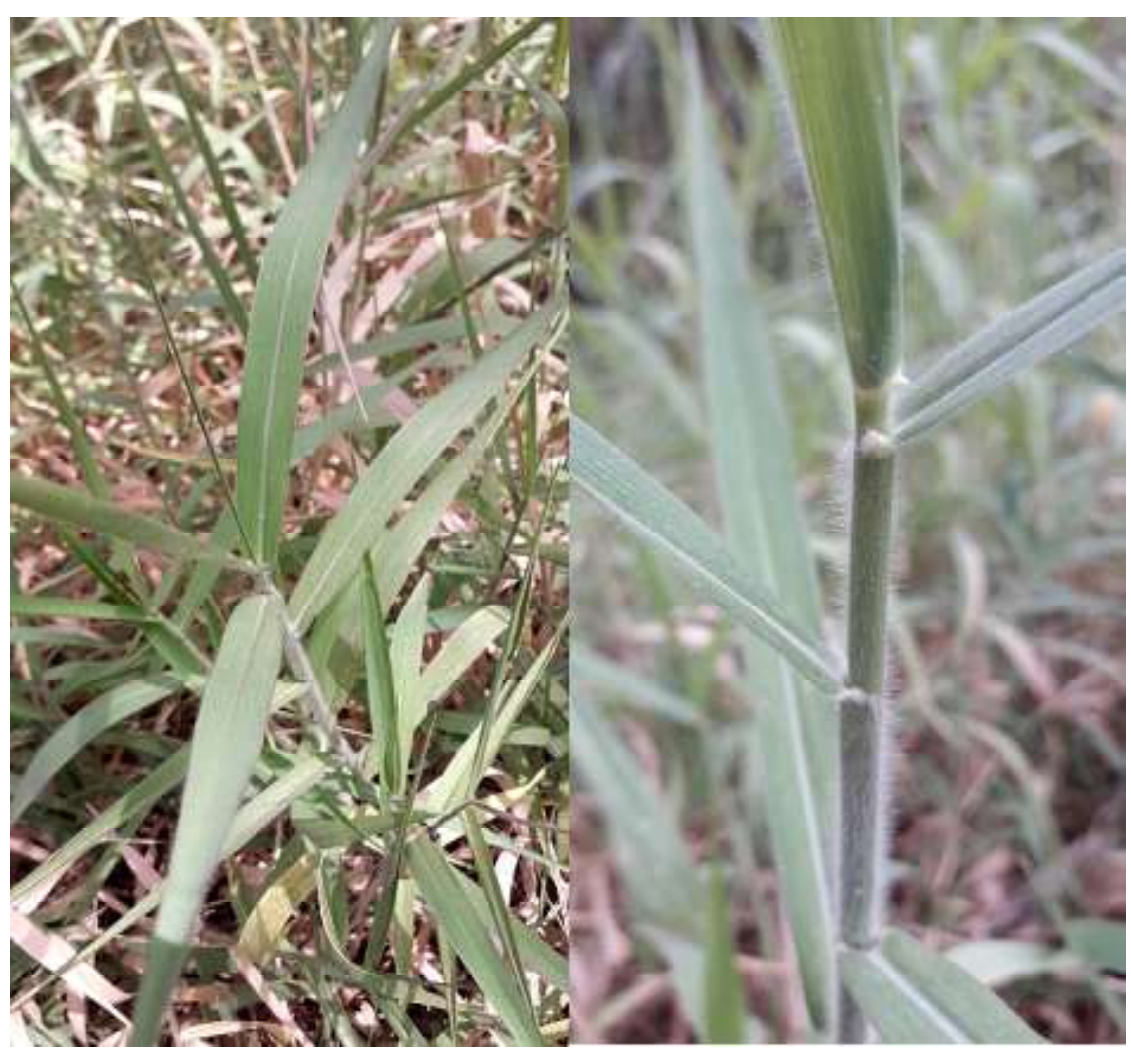

Figura 3. Desarrollo vegetativo de la planta de U. mutica. (Izquierda) Se visualizan estructuras como las hojas y el tallo. (Derecha) Detalle de la pubescencia

tación. Posee niveles de proteína bruta (PB) de aproximadamente 8.9 a $9.4 \%$ cuando la planta es cosechada entre el día 42 al 56 después del rebrote (Alvarado et al., 1990). Según Miles (2006), las características bromatológicas cambian de acuerdo con las condiciones medioambientales bajo las cuales se encuentre el pasto, además del manejo agronómico que reciba. Laiton (2019) reporta valores más elevados de FDN y fibra detergente ácida (FDA) (54.16 y 46.05\%, respectivamente.

\section{Urochloa mutica (Forssk) T.Q. Nguyen}

Gramínea perenne, con estolones extensos y altamente pubescentes. El tallo va de erecto a decumbente y suele ser suave. La vaina posee tricomas en forma de collar. Las hojas son lanceoladas de 10 a $15 \mathrm{~mm}$ de ancho y 25 a $30 \mathrm{~cm}$ de largo (Figura 3). La inflorescencia es en panícula terminal de 20 $\mathrm{cm}$ de largo, con 8 a 20 racimos axilares con espiguillas densas. La inflorescencia posee anteras moradas o amarillas y sus estigmas son morados (Peters y Franco, 2011).

Según Almaraz et al. (2019), el pasto Pará presentó valores de PC de 15.98\%, mientras que Cardona et al. (2002) encontraron valores de $8.17 \%$, además de una FDN de $69 \%$ y una FDA de $41 \%$. Estos valores se encuentran cercanos a los resultados para proteína cruda reportados para el pasto en este trabajo (Cuadro 1). Estos valores de PC pudieron deberse a que el pasto fue analiza- 


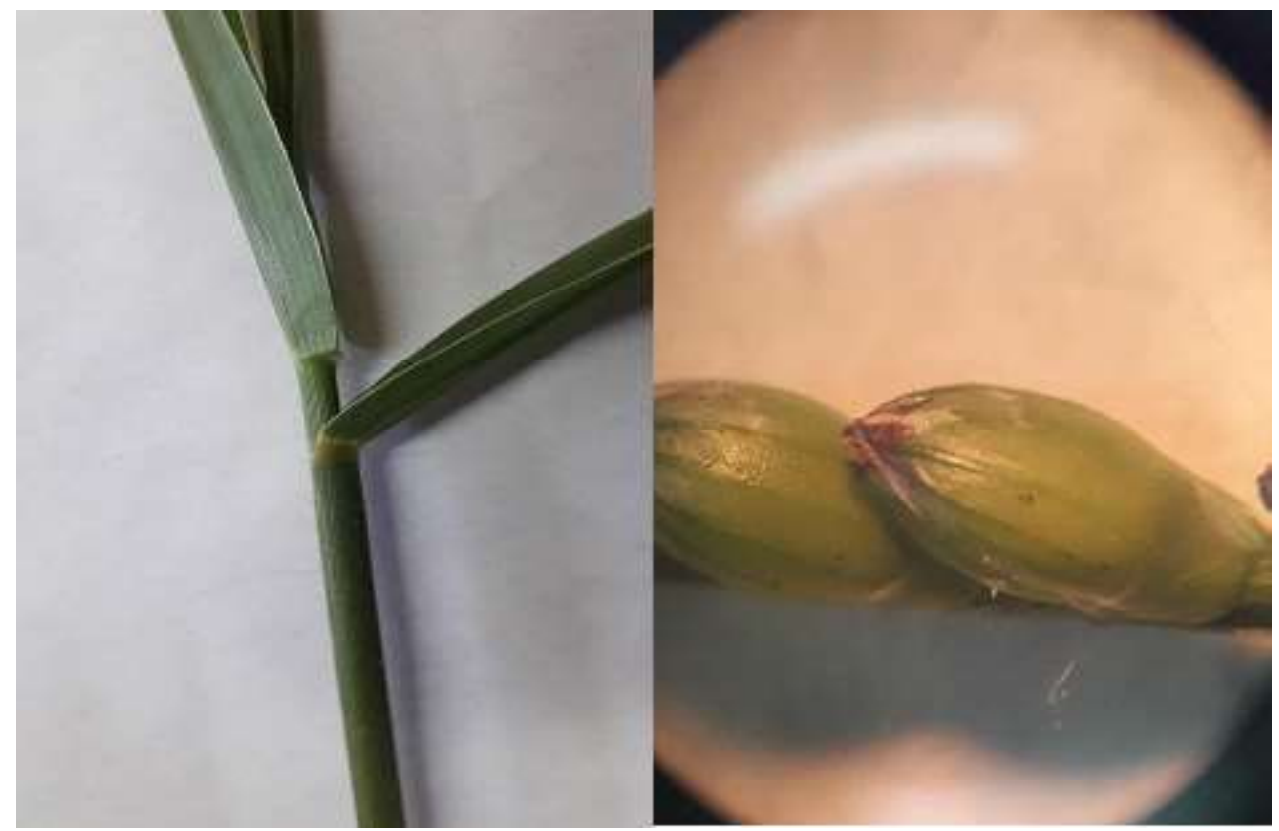

Figura 4. U. brizantha cv Piatá. (Izquierda) Estructuras sin presencia de tricomas. (Derecha) Estructuras morfológicas que componen la espiguilla

do en una época lluviosa, ya que en épocas de menores precipitaciones el contenido de proteína de la $U$. mutica es menor debido a la mayor concentración de material fibroso (López et al., 2018).

Urochloa brizantha (Hochst. ex A. Rich) R. Webster cv Piatá

Cultivar seleccionado de evaluaciones realizadas por la empresa brasileña de investigación agropecuaria EMBRAPA (Peters y Franco, 2011). Es una planta en forma de macolla, de porte medio, con alturas entre $0.85 \mathrm{y}$ $1.10 \mathrm{~m}$ de largo. Las hojas miden hasta $45 \mathrm{~cm}$ de largo y $1.8 \mathrm{~cm}$ de ancho; no poseen tricomas, pero son cortantes en los bordes y la inflorescencia posee hasta 12 racimos florales (Valle et al., 2007) (Figura 4). Las espiguillas son de $7 \mathrm{~mm}$ de largo y tiene una gluma pequeña con aproximadamente 10 nervios; además, las glabras y el ápice de la espiguilla son de color negro (Villalobos y Montiel, 2015).
Según Peters y Franco (2011), el pasto Piatá posee alrededor de $11 \%$ de PC y una digestibilidad aproximada del 60\%. Asimismo, se adapta a suelos de mediana fertilidad y es un pasto de buena persistencia y productividad.

\section{Ajuste de las Curvas de Crecimiento a Ecuaciones Lineales y No Lineales}

Las curvas de crecimiento de las cuatro Urochloas analizadas se presentan en Figura 5. El pasto Pará alcanzó una mayor altura, seguido por el pasto Piatá. Esto, sin embargo, pudo deberse a que los datos de los pastos no se tomaron en el mismo periodo de tiempo y algunos se tomaron en periodos de tiempo más largos. López et al. (2018) encontraron que el pasto Pará (U. mutica) obtuvo una mayor altura, $184 \mathrm{~cm}$ al finalizar su crecimiento. Con respecto a $U$. decumbens, Luna et al. (2015) encontraron que esta especie presenta menor crecimiento $(67.83 \mathrm{~cm})$ 


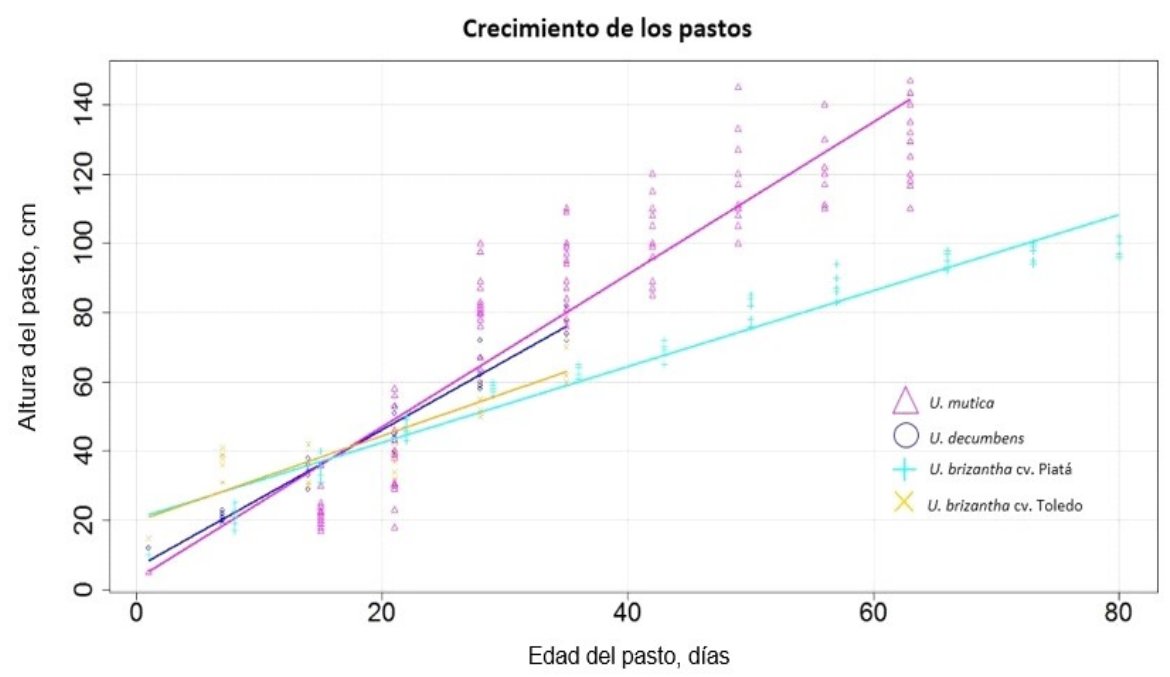

Figura 5. Curvas de crecimiento de Urochloa brizantha (cultivares Toledo y Piatá), Urochloa decumbens y Urochloa mutica

Cuadro 1. Composición nutricional de las cuatro especies (U. brizantha cv Toledo, $U$. decumbens, U. mutica, U. brizantha cv Piatá) respectivamente

\begin{tabular}{lcccc}
\hline & $\begin{array}{c}\text { U. brizanta, } c v \\
\text { Toledo }\end{array}$ & U. decumbens & U. mutica & $\begin{array}{c}\text { U. brazintha } \\
\text { cv Piatá }\end{array}$ \\
\hline Materia seca & 28.9 & 28.9 & 20.5 & 36.5 \\
Proteína bruta & 11.5 & 7.8 & 10.3 & 13.4 \\
Fibra detergente neutra & 65.3 & 35.1 & 73.5 & 65.0 \\
Fibra detergente ácida & 32.1 & 23.8 & 36.7 & 32.0 \\
Lignina & 3.4 & 5.5 & 4.0 & 4.0 \\
Grasa cruda & 6.3 & 2.7 & 2.2 & 2.6 \\
Cenizas & 18.1 & 12.1 & 12.7 & 16.7 \\
Azúcares & 1.2 & 6.8 & 2.7 & 2.4 \\
\hline
\end{tabular}

Análisis efectuado en el en el laboratorio de nutrición animal de la Facultad de Ciencias Agrarias de la Universidad de Antioquia

al compararse con las especies mejoradas de $U$. brizantha $(71.64 \mathrm{~cm})$. En el Cuadro $1 \mathrm{se}$ detallan los resultados del análisis bromatológico realizado a las cuatro Urochloas.

Las alturas alcanzadas por las especies bajo evaluación se presentan en el Cuadro 2. Las desviaciones estándar indican una alta dispersión de los datos. Esto pudo deberse al momento en el que se midieron los datos en campo, dado que la altura de cada planta fue tomada aleatoriamente y no se tuvo una muestra de plantas específica para las mediciones. El resultado de la media arrojada en el pasto Toledo pudo estar influenciado por la baja cantidad de datos recolectados con respecto a las otras especies. La $U$. decumbens 
Cuadro 2. Altura $(\mathrm{cm})$ de las especies $\mathrm{y}$ cultivares de Urochloa (Antioquia, Colombia)

\begin{tabular}{lcc}
\hline Especie & $\mathrm{n}$ & $\begin{array}{c}\text { Media } \pm \mathrm{DE} \\
(\mathrm{cm})\end{array}$ \\
\hline $\begin{array}{l}\text { Urochloa } \\
\text { decumbens }\end{array}$ & 25 & $47.03 \pm 21.31$ \\
$\begin{array}{l}\text { Urochloa mutica } \\
\begin{array}{l}\text { Urochloa } \\
\text { brizantha } \mathrm{cv}\end{array}\end{array}$ & 121 & $88.17 \pm 41.15$ \\
$\begin{array}{l}\text { Piatá } \\
\text { Urochloa } \\
\text { brizantha } \mathrm{cv}\end{array}$ & 55 & $67.46 \pm 26.49$ \\
Toledo & 25 & $44.81 \pm 15.38$ \\
\hline
\end{tabular}

Datos extraídos del análisis estadístico realizado a la base de datos de la altura $(\mathrm{cm})$ de los pastos analizados. La altura fue medida desde la base del tallo hasta la hoja bandera

presenta una media de crecimiento similar al trabajo realizado por Rincón (2011), donde la planta alcanzó una altura máxima de $28 \mathrm{~cm}$ a los 30 días de evaluación en época lluviosa.

En el Cuadro 3 se muestran las medias de la precipitación que se presentaron en el tiempo de las mediciones. Las lluvias más bajas se presentaron para las épocas donde se realizaron las mediciones del pasto $U$. mutica y $U$. brizantha cv Piatá. Los resultados muestran que las plantas que recibieron menor cantidad de precipitación pluvial presentaron mayor crecimiento. Según Peters y Franco (2011), el rango de precipitación para $U$. mutica y $U$. brizantha cv Piatá debe estar entre 1000 a $4000 \mathrm{~mm}$ anuales, lo cual coincide con el promedio de precipitación acumulado encontrado para los pastos en este trabajo. Es decir, la precipitación que tuvieron estas dos especies estuvo en el rango óptimo para su crecimiento.
En el Cuadro 4 se presentan las medias para la variable días con respecto a cada pasto. Es de esperarse que los pastos que tuvieron mayor crecimiento, en este caso la $U$. brizantha cv Piatá y U. mutica obtuvieran una media de días mayor, resultados que concuerdan con el estudio de Garay et al. (2012) con cinco Urochloas donde se obtuvo un

Cuadro 3. Niveles de precipitación pluvial registrados para los cultivos de las especies y cultivares de Urochloa (Antioquia, Colombia)

\begin{tabular}{lc}
\hline Especie & $\begin{array}{c}\text { Media } \pm \mathrm{DE} \\
(\mathrm{mm})\end{array}$ \\
\hline Urochloa decumbens & $10.20 \pm 12.82$ \\
$\begin{array}{l}\text { Urochloa mutica } \\
\text { Urochloa brizantha } \mathrm{cv}\end{array}$ & $4.71 \pm 4.05$ \\
$\begin{array}{l}\text { Piatá } \\
\text { Urochloa brizantha } \mathrm{cv}\end{array}$ & $5.03 \pm 8.82$ \\
Toledo & $6.20 \pm 8.68$ \\
\hline
\end{tabular}

La información fue tomada en los meses que se hizo la evaluación de las pasturas (marzo a junio de 2017 y septiembre a noviembre de 2018 (Fuente: SIATA)

Cuadro 4. Días de crecimiento para los cultivos de las especies y cultivares de Urochloa (Antioquia, Colombia)

\begin{tabular}{lc}
\hline Especie & $\begin{array}{c}\text { Media } \pm \mathrm{DE} \\
(\mathrm{n})\end{array}$ \\
\hline Urochloa decumbens & $20.35 \pm 10.53$ \\
$\begin{array}{l}\text { Urochloa mutica } \\
\text { Urochloa brizantha } \mathrm{cv}\end{array}$ & $39.77 \pm 16.83$ \\
$\begin{array}{l}\text { Piatá } \\
\text { Urochloa brizantha } \mathrm{cv}\end{array}$ & $21.80 \pm 23.50$ \\
Toledo & $20.23 \pm 10.65$ \\
\hline
\end{tabular}


Cuadro 5. Correlación y significancia de las variables días, temperatura y precipitación pluvial para el crecimiento del pasto Pará (Urochloa mutica)

\begin{tabular}{lccc}
\hline & $\begin{array}{c}\text { Altura } \\
(\mathrm{cm})\end{array}$ & $\begin{array}{c}\text { Días } \\
(\mathrm{n})\end{array}$ & $\begin{array}{c}\text { Precipitación } \\
(\mathrm{mm})\end{array}$ \\
\hline Días & $0.94^{* * *}$ & & \\
Precipitación & $0.33^{* * *}$ & $0.29 * *$ & \\
Temperatura & $0.25^{* *}$ & $0.42^{* * *}$ & -0.14 \\
\hline
\end{tabular}

*** $p<0.001 ; * * p<0.01$

Cuadro 6. Correlación y significancia de las variables días, temperatura y precipitación pluvial para el crecimiento del pasto Urochloa decumbens

\begin{tabular}{lccc}
\hline & $\begin{array}{c}\text { Altura } \\
(\mathrm{cm})\end{array}$ & $\begin{array}{c}\text { Días } \\
(\mathrm{n})\end{array}$ & $\begin{array}{c}\text { Precipitación } \\
(\mathrm{mm})\end{array}$ \\
\hline Días & $0.98^{* * *}$ & & \\
Precipitación & $-0.38^{* *}$ & $-0.41^{* *}$ & \\
Temperatura & -0.15 & -0.2 & $-0.48^{* *}$ \\
\hline
\end{tabular}

*** $p<0.001 ; * * p<0.01$

Cuadro 7. Correlación y significancia de las variables días, temperatura y precipitación pluvial para el crecimiento del pasto Piatá (Urochloa brizantha cv Piatá)

\begin{tabular}{lccc}
\hline & $\begin{array}{c}\text { Altura } \\
(\mathrm{cm})\end{array}$ & $\begin{array}{c}\text { Días } \\
(\mathrm{n})\end{array}$ & $\begin{array}{c}\text { Precipitación } \\
(\mathrm{mm})\end{array}$ \\
\hline Días & $0.97^{* * *}$ & & \\
Precipitación & $0.40^{* *}$ & $0.47^{* * *}$ & \\
Temperatura & $-0.32^{* *}$ & $-0.42^{* *}$ & $-0.54 * * *$ \\
\hline
\end{tabular}

*** $p<0.001 ; * * p<0.01$

Cuadro 8. Correlación y significancia de las variables días, temperatura y precipitación pluvial para el crecimiento del pasto Toledo (Urochloa brizantha cv Toledo)

\begin{tabular}{lccc}
\hline & $\begin{array}{c}\text { Altura } \\
(\mathrm{cm})\end{array}$ & $\begin{array}{c}\text { Días } \\
(\mathrm{n})\end{array}$ & $\begin{array}{c}\text { Precipitación } \\
(\mathrm{mm})\end{array}$ \\
\hline Días & $0.85^{* * *}$ & & \\
Precipitación & 0.06 & -0.34 & \\
Temperatura & -0.34 & -0.01 & $-0.86^{* * *}$ \\
\hline
\end{tabular}

*** $p<0.001 ; * * p<0.01$ 

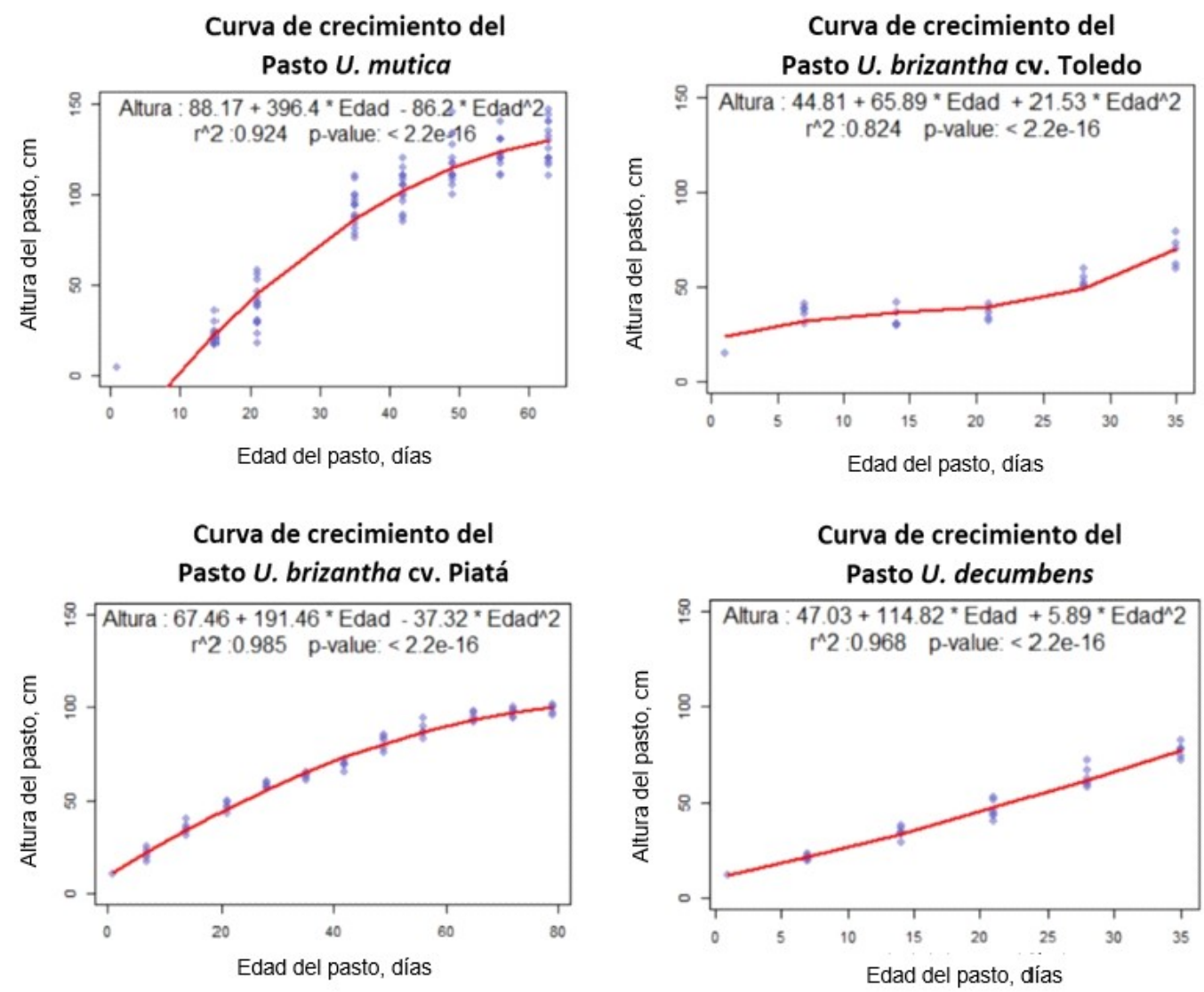

Figura 6. Curvas de crecimiento de las especies de Urochloa ajustadas a un modelo matemático cuadrático. Para cada una de ellas se determinó la ecuación de la recta, el coeficiente de determinación $\left(\mathrm{R}^{2}\right)$ y el p-value

mayor crecimiento en el pasto Piatá desde el inicio de su crecimiento.

La variable temperatura presentó una media de $21.7^{\circ} \mathrm{C}$ para las cuatro especies de pastos evaluadas con una baja desviación estándar $\left( \pm 1.23^{\circ} \mathrm{C}\right)$, lo cual indica que la temperatura se mantuvo constante y sin grandes fluctuaciones durante el periodo de evaluación de las cuatro especies.

\section{Correlaciones}

En el Cuadro 5 se observa una correlación positiva fuerte entre la altura del pasto $U$. mutica con los días de crecimiento, en tanto que con la temperatura ambiental $\left({ }^{\circ} \mathrm{C}\right)$ y la precipitación pluvial $(\mathrm{mm})$ se presentó una correlación positiva débil. Esto puede ser explicado debido a que valores más altos de temperatura y una precipitación adecuada favorecen el proceso de la fotosíntesis para la planta. Según Peters y Franco (2011), $U$. mutica es una especie que crece bien en suelos mal drenados y húmedos y en altitudes entre 0 y $1800 \mathrm{msnm}$.

Existe una correlación positiva fuerte entre los días de crecimiento y la altura de la planta Urochloa decumbens (Cuadro 6). Sin embargo, se encontró una correlación negativa y débil entre la altura de la planta con la 
temperatura $\left({ }^{\circ} \mathrm{C}\right)$ y la precipitación $(\mathrm{mm})$. Esta especie se adapta a zonas donde las precipitaciones anuales están en el rango de 1000 a $3500 \mathrm{~mm}$, pero no resiste suelos mal drenados y no soporta encharcamientos (Peters y Franco, 2011), lo cual explica que a medida que aumenta la precipitación se genera un efecto negativo en el crecimiento de la planta.

Se encontró una correlación positiva fuerte entre los días de crecimiento y la altura de la planta Urochloa brizantha cv Piatá (Cuadro 7). Según Peters y Franco (2011), el crecimiento del pasto Piatá se ve favorecido por precipitaciones anuales superiores a 1000 $\mathrm{mm}$. Además, las condiciones de humedad del suelo durante el periodo de evaluación generaron una correlación positiva sobre el desarrollo de la planta. Valle et al. (2007) afirman que esta especie no soporta periodos de sequía prolongados.

No hubo significancia estadística $(\mathrm{p}>0.05)$ entre las variables temperatura $\left({ }^{\circ} \mathrm{C}\right)$ y precipitación $(\mathrm{mm})$ con respecto a la altura de la planta (Cuadro 8); sin embargo, la correlación entre los días y la altura de crecimiento fue positiva fuerte. Según Argel et al. (2000), esta especie puede alcanzar alturas de hasta $1.60 \mathrm{~m}$. Esta planta posee un tallo hueco, el cual permite adaptarse a zonas inundadas (Gómez, 2017).

\section{Modelos Matemáticos Ajustados a los Datos de Crecimiento}

Se estableció una relación entre el crecimiento y la edad de las cuatro Urochloas evaluadas, mediante un análisis de regresión donde se emplearon modelos lineales y no lineales. También se tuvo en cuenta el coeficiente de determinación $\left(\mathrm{R}^{2}\right)$ y el $\mathrm{p}$-value de la curva. $\mathrm{Al}$ analizar la relación entre la altura y la edad (Figura 6) en las cuatro especies de pastos, los datos se ajustaron a modelos cuadráticos obteniendo la ecuación $y t=\beta_{0}$ $+\beta_{1} t-\beta_{3} t^{2}$, con altos coeficientes de deter- minación y niveles de significancia ( $\mathrm{p}<0.001)$. Las curvas de crecimiento se ajustaron a modelos cuadráticos debido a que no se contaba con suficientes datos a través del tiempo. Según Paine et al. (2012) para mejorar la confiabilidad en la estimación de parámetros se recomienda medir pocos individuos por punto de tiempo y usar muchos puntos de tiempo, se deben tomar muestras de individuos de manera representativa y adherirse a los requisitos de los modelos de efectos mixtos, y además, se deben realizar observaciones con mayor frecuencia en periodos de cambios rápidos en el crecimiento. El coeficiente de determinación $\left(\mathrm{R}^{2}\right)$ muestra a $U$. brizantha cv Piatá $\left(\mathrm{R}^{2}=0.9859\right)$ como la especie que se ve más influenciada positivamente en su crecimiento por los días de evaluación.

Una causa que puede explicar la razón por la cual las curvas no se ajustaron a los modelos no lineales (Gompertz, Verhulst, Brody, Von Bertalanffy, Richards), es precisamente debido a los pocos datos tomados en un tiempo tan corto, los cuales no describen una curva sigmoidea, la que es una característica de estos modelos al ajustarse a la curva de crecimiento de seres vivos. Esta afirmación se puede comparar con el estudio de Rodríguez et al. (2011), donde se evaluó el crecimiento del pasto Pennisetum purpureum cv Cuba CT- 169 y gracias a las repeticiones en el tiempo, 11 periodos de observaciones en 140 días de medición, las curvas se ajustaron a modelos matemáticos lineales simples, no lineales logísticos y exponenciales. Aspecto que se puede apreciar en la curva de $U$. brizantha cv Piatá (Figura 6) que muestra un comportamiento curvo, mas no sigmoideo, pero que sus observaciones en el tiempo son cortas al solo evaluar el pasto durante 80 días de vida. Jenkins y Quintana-Ascencio (2020) recomiendan utilizar muestras de datos superiores a 25 para evitar problemas de falta de ajuste en modelos de regresión. 


\section{Conclusiones}

- Las curvas de crecimiento de las Urochloas se ajustaron a modelos cuadráticos que permitieron la comparación entre ellas.

- $U$. brizantha cv Piatá $\left(\mathrm{R}^{2}=0.9859\right)$ fue la especie más influenciada positivamente en su crecimiento por efecto de los días de edad, seguida de $U$. decumbens $\left(\mathrm{R}^{2}=0.968\right)$, U. mutica $\left(\mathrm{R}^{2}=0.924\right)$ y $U$. brizantha cv Toledo $\left(\mathrm{R}^{2}=0.824\right)$.

- El crecimiento de las Urochloas depende y puede verse afectado por las variables climáticas de temperatura y precipitación, además de otras condiciones y características edafoclimáticas y de manejo.

- Los registros fotográficos de las Urochloas permitieron diferenciar las especies con base en sus estructuras morfológicas.

\section{Literatura Citada}

1. Agudelo D, Cerón M, Restrepo L. 2008. Modelación de las funciones de crecimiento aplicadas a la producción animal. Rev Colomb Cienc Pec 21:39-58.

2. Almaraz L, García A M, Sánchez, , Torres N, Herrera J, Bolttini MB, Rojas AR. 2019. Análisis bromatológico y producción de gas in vitro de forrajes utilizaos en el trópico seco mexicano. Arch Zootec 68: 260-266. doi: 10.21071/ az.v68i262.4145

3. Alvarado A, Arriojas L, Chacón E, Rodríguez, S, Chacín F. 1990. Estudios sobre henificación del pasto Barrera (Brachiaria decumbens Stapf) en condiciones de sabanas del Piedemonte Barinés I. Producción y valor nutritivo de la materia seca. Zootec Trop 8: 17-36.

4. Argel P, Hidalgo C, Lobo M. 2000. Pasto Toledo (Brachiaria brizantha ClAT 26 110). Gramínea de crecimiento vigoroso con amplio rango de adaptación a condiciones de trópico húmedo y subhúmedo. Boletín Técnico. San José, Costa Rica: Ministerio de Agricultura y Ganadería de Costa Rica. 15 p.

5. Argel P, Keller-Grein G, Centro Internacional de Agricultura Tropical (CIAT). 1998. Experiencia regional con Brachiaria: región de América Tropical, tierras bajas húmedas. Brachiaria: biología, agronomía y mejoramiento. Colombia: CIAT. 22 p.

6. Brown J, Fitzhugh H, Cartwright T. 1976. Comparison of nonlinear models for describing weight-age relationships in cattle. J Anim Sci 42: 810-818. doi: 10.2527/jas1976.424810x

7. Cardona M, Sorza JD, Posada SL, Carmona JC, Ayala SA, Alvarez OL. 2002. Establecimiento de una base de datos para la elaboración de tablas de contenido nutricional de alimentos para animales. Rev Colomb Cienc Pec 5: 240-246

8. [CIAT] Centro Internacional de Agricultura Tropical. 2010. Ganado, cambio climático y Brachiaria. Hoja informativa $\mathrm{N}^{\mathrm{o}} 12$. [Internet]. Disponible en: https://ciat.cgiar.org/

9. Combatt E, Jarma A, \& Paternina E. 2015. Bromatología de Brachiaria decumbens Stapf y Cynodon nlemfuensis Vanderyst en suelos sulfatados ácidos en Córdoba, Colombia. Rev Mex Cienc Agríc 6: 1035-1049.

10. Freitas A. 2005. Curvas de crescimento na produção animal. Rev Bras Zootecnç 34: 786. doi: 10.1590/ S1516-35982005000300010

11. Garay J, Cienfuegos J, Espinosa P, Zárate, M, Ibarra. 2012. Análisis de crecimiento inicial de cinco genotipos de Bracharia en trópico húmedo. Tsafiqui Rev Invest Cient. 3: 83-93. doi: 10.29019/tsafiqui.v0i3.223

12. Giraldo D. 2013. Las gramíneas en Colombia. Riqueza, distribución, endemismo, invasión, migración, usos y taxonomías populares. Colombia: Univ. Nacional de Colombia. 384 p. 
13. Gómez M. 2017. Cultura empresarial ganadera. Catálogo de gramíneas de 2017. [Internet]. Disponible en: https:// culturaempre-sarialganadera.-files.wordpress.com/2017/01/ftbrachiariaarrectaceg-2017.pdf

14. Hunt R. 1978. Demography versus plant growth analysis. New Phytol 80: 269-272.

15. Hunt R, Thomas B, Murphy D J, Murray D. 2003. Growth analysis, individual plants. Encyclopedia of Applied Plant Sciences 2: 579-588. doi: 10.1016/ B978-0-12-394807-6.00226-4

16. Jenkins DG, Quintana-Ascencio PF. 2020. A solution to minimum sample size for regressions. Plos One 15: e 0229345. doi: 10.1371/journal.pone.0229345

17. Laiton JF. 2019. Evaluación de tres especies de Brachiaria spp, bajo métodos de pastoreo rotacional, en sabanas del piedemonte del municipio de Tame, Arauca. Tesis de Maestría. Villavicencio, Meta-Colombia. $70 \mathrm{p}$.

18. López A, Nuñez D, Jimny, Aguirre T, Lucrecia, Flores M, Enrique. 2018. Dinámica de la producción primaria y valor nutritivo de tres gramíneas tropicales (Melinis minutiflora, Setaria sphacelata y Brachiaria mutica) en tres estados fenológicos. Rev Invest Vet Perú 29: 396-409. doi: 10.15381/rivep.v29i2.14494

19. Lascano C, Pérez R, Plazas B, Camilo H, Medrano J, Argel M, Pedro J. 2002. Pasto Toledo (Brachiaria brizantha CIAT 26110): gramínea de crecimiento vigoroso para intensificar la ganadería colombiana. Cali, Colombia: Centro Internacional de Agricultura Tropical. $18 \mathrm{p}$.

20. Luna R, Espinoza A, Avellaneda J, Reyes Pérez J, Luna M, Iza N. 2016. Respuesta agronómica de tres variedades de Brachiaria en el cantón El Empalme provincia del Guayas, Ecuador. Ciencia Tecnol 8: 45-50.
21. Miles, J. 2006. Mejoramiento genético en Brachiaria. Objetivos estratégicos, logros y proyección. Pasturas Tropicales 28: 26-30.

22. Mohr S. 1995. Plant physiology. Berlin: Springer. $629 \mathrm{p}$.

23. Olivera Y, Machado R, Pozo P. 2006. Características botánicas y agronómicas de especies forrajeras importantes del género Brachiaria. Pastos y Forrajes 29: 1-23.

24. Ortega CA, Lemus C, Bugarín JO, Alejo G, Ramos A, Grageola $O$, Bonilla JA. 2015. Características agronómicas, composición bromatoló-gica, digestibilidad y consumo animal en cuatro especies de pastos de los géneros Brachiaria y Panicum. Agroecos Trop Subtrop 18: 291-301.

25. Paine CET, Marthews TR, Vogt DR, Purves D, Rees M, Hector A, Turnbull LA. 2012. How to fit nonlinear plant growth models and calculate growth rates: an update for ecologists. Methods Ecol Evol 3: 245-256. doi: 10.1111/j.2041210X.2011.00155.X

26. Peters M, Franco L. 2011. Especies forrajeras multipropósito opciones para productores del trópico americano. Colombia: CIAT. 222 p.

27. Pinheiro J, Bates D. 2000. Mixed effects models in S and S-PLUS. Berlin: Springer-Verlag. $528 \mathrm{p}$.

28. Ramírez JL, Herrera RS, Leonard I, Cisneros M, Vardecia D, Álvarez Y. 2011. Relación entre factores climáticos, rendimiento y calidad de Pennisetum purpureum vc. Cuba CT 169 en el Valle del Cauto, Cuba. Rev Cubana Cien Agríc 45:293-297.

29. Rodríguez L, Torres V, Martínez R, Jay O, Noda A, Herrera M. 2011. Modelos para estimar la dinámica de crecimiento de Pennisetum purpureum vc. Cuba CT169. Cuban J Agr Sci 45: 349-354. 
30. Renvoize S. 1987. A survey of leafblade anatomy in grasses XI. Paniceae. Kew Bull 42: 739-768.

31. Rincón A. 2011. Efecto de alturas de corte sobre la producción de forraje de Brachiaria sp en el piedemonte llanero de Colombia. Corpoica 12: 107-112.

32. Rivas L, Holmann F. 2004. Impacto económico potencial de nuevos híbridos de Brachiaria resistentes al salivazo: Llanos orientales y costa norte de Colombia. Cali, Colombia: CIAT. 38 p.

33. Torres V, Barbosa I, Meyer R, Noda A. Sarduy L. 2012. Criterios de bondad de ajuste en la selección de modelos no lineales en la descripción de compor- tamientos biológicos. Cuban J Agr Sci 46: 345-350.

34. Valle C, Euclides V, Valério J, Macedo M, Fernandes C, Dias-Filho M. 2007. Brachiaria brizantha cv. Piatã: umaforrageira para diversifcação de pastagens tropicais. Seed News 11: 28-30.

35. Vanegas LÁ. 2015. Dinámica de crecimiento y criterios de pastoreo para optimizar el uso de praderas Brachiaria brizantha cv Toledo. Tesis de Maestría. Bogotá, Colombia: Univ. de la Salle. 253 p.

36. Villalobos L, Montiel M. 2015. Características taxonómicas de pastos Brachiaria utilizados en Costa Rica. Nutr Anim Trop 9: 39-56. 